donation, survival after the diagnosis of brain death and length of stay in PICU $(\mathrm{p}>0.05)$.

Conclusion The early diagnosis of brain death and prompt evaluation of the patients by the ICU physicians once the diagnosis is considered will probably yield better organs and reduce costs. Training of the PICU physicians, nurses and organ donation coordinators and increasing the awareness of the children via public means may increase the families' acceptance rate for organ donation in future.

\section{PO-0292 PAEDIATRIC OUT-OF-HOSPITAL PATIENTS: A POPULATION-BASED STUDY}

${ }^{1} \underline{\mathrm{H} \text { Salmi, }}{ }^{2} \mathrm{H}$ Harve-Rytsälä, ${ }^{2} \mathrm{M}$ Kuisma. ${ }^{1}$ Department of Anesthesia and Intensive Care, HUCH Children's Hospital, Helsinki, Finland; ' ${ }^{2}$ Department of Prehospital and Emergency Care, Helsinki University Central Hospital, Helsinki, Finland

\subsection{6/archdischild-2014-307384.944}

Background and aims The incidence and distribution of paediatric out-of-hospital $(\mathrm{OOH})$ emergencies are not known, and the need for paediatric $\mathrm{OOH}$ services has not been studied on a population level. We wanted to study the characteristics and epidemiology of paediatric $\mathrm{OOH}$ care. We hypothesised this could ameliorate the design of paediatric emergency medical services (EMS) and the education of their personnel.

Methods We studied all $(\mathrm{n}=1869)$ dispatched emergency calls and the connected EMS patient records for paediatric patients (age 0-16 y) in Helsinki, Finland (population 0.6 M, paediatric population 91000 ) during a 12-month period (2012). Patient characteristics, diagnoses, time intervals, medical treatments, procedures, vital measurements and outcome of $\mathrm{OOH}$ treatment were available for analysis.

Results Paediatric OOH emergencies were relatively rare (1869 calls, or $4.5 \%$ of all emergency calls; yearly incidence 20:1000 in the population). Toddlers were frequently involved, as a third of patients were 0-2 y old. Three causes (falls, dyspnoea, seizures) made up nearly half (37\%) of all paediatric emergencies, and the majority $(80 \%)$ concerned previously healthy children. After evaluation by the EMS, only half of the patients $(54 \%)$ needed ambulance transportation to hospital. Cardiac arrest, or need for advanced life support measures (e.g. intubation), were rarities.

Conclusions Paediatric $\mathrm{OOH}$ emergencies are rare and have specific characteristics differing from the adult population. EMS should be designed and their personnel trained for evaluation and management of most frequent situations.

\section{PO-0293 MANAGEMENT OF CEREBRAL ARTERIOVENOUS MALFORMATION WITH HAEMORRHAGE}

${ }^{1}$ A Scheibl, ${ }^{2} \mathrm{M}$ Tisdall, ${ }^{1} \mathrm{Q}$ Mok. ${ }^{1} \mathrm{PICU}$, Great Ormond Street Hospital for Children NHS Foundation Trust, London, UK; ${ }^{2}$ Neurosurgery, Great Ormond Street Hospital for Children NHS Foundation Trust, London, UK

\subsection{6/archdischild-2014-307384.945}

Background and aims We retrospectively analysed cases of cerebral haemorrhage from arteriovenous malformation (AVM), admitted to a PICU of a tertiary hospital to determine management strategies and outcome.

Methods Data were collected retrospectively from a departmental database between 2009 to 2014 .
Results 10 patients, all previously fit and well presented with spontaneous cerebral haemorrhage in the context of previously undiagnosed AVM. Median age at diagnosis was 12,5 years (815 years). (7 male, 3 female)

All patients presented with headache and decrease in Glasgow coma scale.

All patients had CT scan and angiogram as part of their initial evaluation and MRI for follow-up. Intracranial pressure monitoring devices were inserted in all patients and 5 required emergency craniotomy for haemorrhage evacuation. 6 required embolisation and 4 underwent gamma knife stereotactic radio surgery.

5 patients needed inotropic support with nor adrenaline during the first 24-36 h, 6 developed arterial hypertension during their PICU stay.

Median length of stay in PICU 9,5 days (3-19 days). Median length of invasive ventilation 7 days (2-16 days), 1 patient received a tracheostomy after several failed extubation trials.

Follow up after six months - one year showed seven patients with improving neurological signs, mostly hemiparesis, ataxia and diplopía. Two were asymptomatic and one patient has not reached the six month follow-up yet.

Conclusions Treatment of cerebral haemorrhage in context of arteriovenous malformation includes a multidisciplinary approach with satisfactory results in neurological outcome on follow-up.

\section{PO-0294 WITHDRAWN}

\section{PO-0295 EARLY NEONATAL HYPOCALCEMIA IN INFANTS BORN TO MOTHERS WITH HIGH PREVALENCE OF HYPOVITAMINOSIS D AND RELATION TO BIRTH SIZE}

A Soliman, E Shatlah, A Adel. Pediatrics, Hamad Medical Centre, Doha, Qatar

\subsection{6/archdischild-2014-307384.946}

Introduction Neonatal hypocalcemia is a potentially life-threatening condition, with reported prevalence varying by gestational age, maternal and infant comorbidities, and perinatal factors.

Objectives Because of the high prevalence of vitamin D deficiency in pregnant women $(60 \%)$ in Qatar we measured serum $\mathrm{Ca}$ in a random sample of newborns admitted to the neonatal unit in HMC for 6 consecutive months and correlated seum calcium level to gestational age, birth weight, length and head circumference, and mothers' weight.

Results (Table) : The incidence of hypocalcemia in our preterm and full term neonates was significantly higher (3\%) compared to those reported in the literature. There was no statistical difference in the incidence of hypocalcemia between term and preterm groups. Serum Ca was correlated significantly with birth wt, length, BMI and head circumference $(r=0.26,0.25,0.32$, 0.25 respectively, $\mathrm{p}<0.05)$.

Discussion Despite high prevalence of vitamin D deficiency in pregnant women in Qatar (60\%) early neonatal hypocalcemia was detected only in 3\% of both term and preterm newborns. These findings point out to a well-functioning adaptation mechanism that prevents hypocalcemia in newborns to mothers with hypovitaminosis D.

Conclusion This high incidence of neonatal hypocalcemia necessitates vitamin D supplementation to pregnant women in this population with high prevalence of vitamin D deficiency. 